\title{
SN 1998bw and Other Hyperenergetic Type Ic Supernovae
}

\author{
Paolo A. Mazzali ${ }^{1,2}$, Ken'ichi Nomoto ${ }^{2}$, Jinsong Deng ${ }^{2}$, Keiichi Maeda ${ }^{2}$, \\ Koichi Iwamoto ${ }^{3}$, Alexei V. Filippenko ${ }^{4}$, and Ryan T. Foley ${ }^{4}$ \\ 1 INAF - Osservatorio Astronomico di Trieste, Via Tiepolo, 11, Trieste, Italy \\ mazzali@ts.astro.it \\ 2 Department of Astronomy and Research Center for the Early Universe, School \\ of Science, The University of Tokyo, Tokyo 113-0033, Japan \\ 3 Department of Physics, College of Science and Technology, Nihon University, \\ Tokyo 101-8308, Japan \\ 4 Astronomy Department, Univ. of California, Berkeley, CA 94720-3411
}

Summary. The properties of the best-observed peculiar, SN $1998 \mathrm{bw}-$ like Type Ic supernovae (sometimes called "hypernovae") are reviewed, starting from SN 1998bw itself and including SNe 1997ef and 2002ap. Analysis of the light curves and the spectra shows that, while these SNe display a range of properties (kinetic energy, mass of the ejecta, mass of ${ }^{56} \mathrm{Ni}$ synthesized in the explosion), they have in common a larger-than-normal explosion kinetic energy, giving rise to the characteristic broad-lined spectra. Also, they all come from the collapse of bare CO cores of massive $\left(\gtrsim 20 \mathrm{M}_{\odot}\right.$ ) progenitor stars. Some of the properties of these SNe, such as kinetic energy and mass of ${ }^{56} \mathrm{Ni}$, are probably correlated with the mass of the progenitor. Evidence that these powerful events are intrinsically asymmetric, suggesting that a correlation with at least some gamma-ray bursts can be expected, is also discussed.

\section{Introduction}

One of the most interesting recent developments in the study of supernovae (SNe) is the discovery of objects with a kinetic energy $\left(E_{\text {kin }}\right)$ on the order of $10^{52} \mathrm{erg}$, about 10 times more than normal core-collapse SNe (hereafter $\left.E_{51}=E / 10^{51} \mathrm{erg}\right)$. The most luminous and powerful of these objects, the Type Ic supernova (SN Ic) 1998bw, was probably linked to the gamma-ray burst GRB 980425 [6]. Moreover, SN 1998bw was exceptional for a SN Ic: it was as luminous at peak as a SN Ia, indicating that it synthesized $\sim 0.5$ $\mathrm{M}_{\odot}$ of ${ }^{56} \mathrm{Ni}$, and it showed very broad absorption lines, reaching velocities of $\sim 30,000 \mathrm{~km} \mathrm{~s}^{-1}$. This was interpreted as due to a very powerful explosion, with $E_{51} \sim 30$ in spherical symmetry $[11,44]$.

Subsequently, other unusually energetic SNe Ic have been discovered or recognized, such as SN 1997ef [12, 23], SN 1997dq [21], possibly SN 1999as [17], and SN 2002ap [26]. These objects did not appear to be associated with GRBs. Fig. 1 shows the near-maximum spectra and the absolute $V$-band light curves of these so-called "hypernovae" and of the normal SN Ic 1994I; they span a wide range of properties. Not all of them are extremely luminous, 

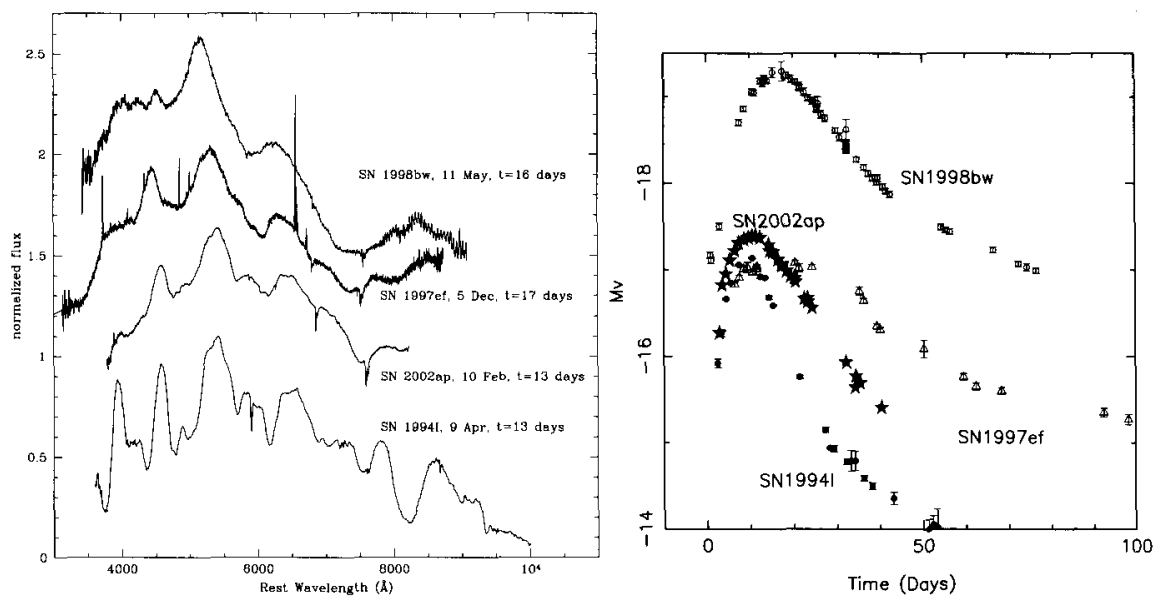

Fig. 1. (a) The near-maximum spectra and (b) the absolute $V$-band light curves of SN Ic 1994I and a few hyperenergetic Type Ic SNe [26].

yet they are spectrally similar to SN $1998 \mathrm{bw}$, so they have also been dubbed "SN 1998bw-like SNe."

In this paper, we examine the properties of SN 1998bw-like SNe as derived from optical light curves and spectra, and we discuss what may be the discriminating factor for their birth or the connection with a GRB.

\section{SN 1998bw and GRB 980425}

The discovery of SN 1998bw immediately generated a great deal of interest, since the SN was detected in the error box of GRB 980425, and was very possibly linked to it. The object was identified as a supernova from its light curve, which was different from those of typical optical transients of GRBs. Extensive data were collected at $\operatorname{ESO}[6,36]$ and other observatories.

Early-time spectra were rather blue and featureless, quite unlike those of other known SNe. More careful inspection showed clear similarities with the spectra of SNe Ic, but with one major difference: the absorption lines were so broad in SN 1998bw that they blended together, giving rise to what could even be confused with an emission spectrum. Very large expansion velocities $\left(\sim 30,000 \mathrm{~km} \mathrm{~s}^{-1}\right)$ were measured in the ejecta. Also, SN $1998 \mathrm{bw}$ was very bright for a SN Ic: for a redshift distance of $39 \mathrm{Mpc}(z=0.0085$ and $H_{0}=65 \mathrm{~km} \mathrm{~s}^{-1} \mathrm{Mpc}^{-1}$ ), the $\mathrm{SN}$ reached a maximum $V=-19.4 \mathrm{mag}$, which is luminous even for a SN Ia, while a typical SN Ic like SN 1994 I was almost 2 mag dimmer [33, 37].

Modeling of the early data ([11], hereafter IMN98) led to several striking conclusions. First, the $\mathrm{SN}$ produced about $0.7 \mathrm{M}_{\odot}$ of ${ }^{56} \mathrm{Ni}$, as much as a SN Ia. 
This was necessary to power the light curve via the deposition of the $\gamma$-rays emitted in the decay of ${ }^{56} \mathrm{Ni}$ into ${ }^{56} \mathrm{Co}$ and ${ }^{56} \mathrm{Fe}$.

IMN98 discuss that the light curves are degenerate: different combinations of ejecta mass $M_{\mathrm{ej}}$ and kinetic energy $E_{\text {kin }}$ can reproduce the data equally well. Spectra came to the rescue: spectral synthesis showed that the only way to reproduce the observed extensive line blending was to have significant amounts of material moving at very large velocities. Since models with different $E_{\text {kin }}$ give different degrees of blending, it was possible to select as the best model one with $M_{\mathrm{ej}}=10.9 \mathrm{M}_{\odot}$ and $E_{51}=30$. This had an exploding core mass of $13.8 \mathrm{M}_{\odot}$ and was designated CO138 accordingly. A typical SN Ic has $M_{\mathrm{ej}}=1 \mathrm{M}_{\odot}$, and the typical kinetic energy of most SNe of all types is only about $E_{51}=1$. An even larger value, $E_{51}=50$ [30], gives a better fit to the declining part of the light curve and to the spectra. A comparable value was obtained from an analysis of the early-time spectra [2]. Because of its exceptionally large $E_{\text {kin }}$, SN 1998bw was called a "hypernova" by some authors [13].

The progenitor must have been a massive star, with a main-sequence mass of about $40 \mathrm{M}_{\odot}$. Also, the remnant mass, which is computed by allowing only as much of the ${ }^{56} \mathrm{Ni}$ produced by core $\mathrm{Si}$ burning to be ejected as is necessary to power the $\mathrm{SN}$ light curve, turned out to be $\sim 3 \mathrm{M}_{\odot}[30,31]$. This probably exceeds the maximum mass of a neutron star, suggesting that the explosion that was observed as SN 1998bw resulted in the creation of a black hole.

Since SN 1998bw was probably connected to a highly non-spherical event like a GRB, departure from spherical symmetry could be expected. Early polarization measurements confirmed this. Polarization of $\sim 0.5 \%$, possibly decreasing with time, was detected $[11,15,36]$. This was interpreted as an axis ratio of about 2:1 in the expanding ejecta [10]. Models of the collapse of a rotating stripped core of a massive star confirmed that the explosion should be asymmetric, thus establishing a possible link with the GRB [19].

The transition to the nebular phase occurred $\sim 100$ days after the outburst, which is assumed to coincide with GRB 980425 . Nebular spectra probe the deepest parts of the ejecta. Early in the nebular epoch the spectra show the properties of a typical SN Ic, with strong lines of [O I], Ca II, [Ca II], and $\mathrm{Mg} \mathrm{I}]$, and weaker lines of [ $\mathrm{Fe} \mathrm{II}]$. However, the [Fe II] lines are unusually strong for a SN Ic. Also, lines of different elements have different widths, indicating different expansion velocities. In particular, iron appears to expand more rapidly than oxygen. Furthermore, the $[\mathrm{O} \mathrm{I}]$ lines decline more slowly than the [Fe II] ones, signaling deposition of $\gamma$-rays in a slowly moving $\mathrm{O}$-dominated region. These facts suggest that the explosion was aspherical. The absence of [Fe III] lines can be understood if the ejecta are significantly clumped.

The properties of aspherical hypernova explosions were computed by Maeda et al. [20]. In such explosions Fe is mostly ejected at high velocity in a jet along the polar direction, while nearer the equatorial plane burning 
is less effective and low-velocity $\mathrm{O}$ is mostly ejected. The unusual ratio of the width of the $\mathrm{O}$ and $\mathrm{Fe}$ nebular lines in SN 1998bw can be explained with a strongly aspherical explosion model viewed from a near-jet direction. Also, in this case the $\mathrm{O}$ line has a very sharp peak, in agreement with the observations. The value of $E_{\text {kin }}$ in these models goes down to $E_{51} \approx 10$.

\section{SNe 1997ef and 1997dq}

SN 1997ef was a SN Ic whose $E_{\text {kin }}$ was determined to be large by both Branch [2], who found $E_{51} \approx 30$ from spectral fits, and Iwamoto et al. [12], who derived from a simultaneous study of the light curve and spectra the values $E_{51}=8, M_{\mathrm{ej}}=8 \mathrm{M}_{\odot}$, and $\mathrm{M}\left({ }^{56} \mathrm{Ni}\right)=0.15 \mathrm{M}_{\odot}$, and suggested that it was the result of the collapse of the stripped $\mathrm{CO}$ core of a massive star $\left(\mathrm{ZAMS} \approx 25-30 \mathrm{M}_{\odot}\right)$. Following the association between $\mathrm{SN} 1998 \mathrm{bw}$ and GRB 980425, Wang et al. [42] looked for other possible associations between $\mathrm{SNe}$ and GRBs, and suggested that GRB 971115 may be compatible with SN 1997ef in position and time of occurrence, although the correlation is much weaker than for SN 1998bw and GRB 980425.

Mazzali et al. [23] analyzed a series of early-time spectra of SN 1997ef. Using spectroscopic dating of the 1997 Nov. 29 spectrum they estimated that the explosion occurred on Nov. $20 \pm 1$ day. They derived the density and abundance distributions in the ejecta, and found that typical hypernova abundances describe SN 1997ef very well. However, their results differed from the explosion model in two ways: (1) the outer density structure turned out to be rather flat (a power law with index $n=-4$ ), as required by the very broad absorption features, extending to $v \approx 25,000 \mathrm{~km} \mathrm{~s}^{-1}$; and (2) matter was found to be present at low velocities $\left(\sim 2,000 \mathrm{~km} \mathrm{~s}^{-1}\right)$, well below the low-velocity cutoff of a spherically symmetric explosion model $\left(5,000 \mathrm{~km} \mathrm{~s}^{-1}\right)$. They found that a model with $E_{51}=20, M_{\mathrm{ej}}=10 \mathrm{M}_{\odot}$, and $M\left({ }^{56} \mathrm{Ni}\right)=$ $0.13 \mathrm{M}_{\odot}$ (model $\mathrm{CO} 100$ ) gives a good representation of the light curve and spectra.

A synthetic light curve obtained with this modified density structure fits the observations near the peak quite nicely. Mazzali et al. [23] suggested that if an additional $0.05 \mathrm{M}_{\odot}$ of ${ }^{56} \mathrm{Ni}$ is contained at low velocities and high density, then the observed slowing down of the light curve between days 60 and 160 might be explained by the ensuing additional deposition of $\gamma$-rays. They also suggested that the distribution of ${ }^{56} \mathrm{Ni}$ may be aspherical.

Matheson et al. [21] showed a set of spectra of the Type Ic SN 1997dq, and suggested that it is similar to SN 1997ef (cf. their Figures 17 and 18). Unlike SN 1997 ef, a nebular spectrum is available for SN 1997dq. This can be used to determine the mass of ${ }^{56} \mathrm{Ni}$ ejected by this SN and, by analogy, by SN 1997ef. We modeled this spectrum with our NLTE nebular code (e.g., [25]). Given the relative distance modulus of SN $1997 \mathrm{dq}$ with respect to SN $1997 \mathrm{ef}(-1.8$ mag), the best fit required $M\left({ }^{56} \mathrm{Ni}\right)=0.16 \mathrm{M}_{\odot}$, and a total mass of $2.3 \mathrm{M}_{\odot}$, 
of which only about $0.8 \mathrm{M}_{\odot}$ consists of $\mathrm{O}$; the rest is mostly $\mathrm{Si}, \mathrm{C}$, and $\mathrm{S}$ [28]. The masses of $\mathrm{Ca}$ and $\mathrm{Mg}$ are rather small by comparison, even though they do give rise to rather strong emission features. The outer velocity of the nebula is only $5,000 \mathrm{~km} \mathrm{~s}^{-1}$, which is similar to the velocity of the nebular component in earlier spectra of SN $1997 \mathrm{ef}$. The density of the outer regions is too low at these advanced epochs to contribute significantly to the nebular spectrum, and the deposition of $\gamma$-rays and positrons is also probably quite small, because ${ }^{56} \mathrm{Ni}$ is not expected to be mostly located there. The fact that the velocities of the regions that form the nebular spectrum are low confirms the finding that significant amounts of mass are present at velocities lower than the cutoff imposed by one-dimensional explosion models [23].

Indeed, Mazzali et al. [23] estimated that the mass between 3,000 and $5,000 \mathrm{~km} \mathrm{~s}^{-1}$ in SN $1997 \mathrm{ef}$ is $1.65 \mathrm{M}_{\odot}$, in good agreement with the result for SN $1997 \mathrm{dq}$, which refers to all regions with $v<5000 \mathrm{~km} \mathrm{~s}^{-1}$. The estimate of the ${ }^{56} \mathrm{Ni}$ mass for SN $1997 \mathrm{dq}$ is also in good agreement with the results obtained for SN 1997ef. Although the nebular spectrum of SN 1997dq is not of exceptionally high signal-to-noise ratio, the line profiles do not seem to show signs of an asymmetric explosion, such as the multiple components (narrow and broad) observed in the nebular spectra of SN 1998bw [25].

\section{SN 2002ap}

SN Ic 2002ap was discovered in M74 on 2002 January 30 [9]. The SN was immediately recognized as SN 1998 bw-like from its broad spectral features $[5,7,16,29]$. Luckily, the SN was discovered very soon after it exploded: the discovery date was Jan. 29, while the SN was not detected on Jan. 25 [32]. This is among the earliest any SN has been observed.

SN 2002ap reached $V$ maximum on about Feb. 8 at $V=12.3$ mag. It peaked earlier than both SNe 1998bw and 1997ef, but later than the normal SN 1994I, suggesting an intermediate value of the ejecta mass $M_{\mathrm{ej}}$. Using a distance to M74 of $8 \mathrm{Mpc}(\mu=29.5 \mathrm{mag}$; [38]), and a combined Galaxy and M74 reddening of $E(B-V)=0.09 \mathrm{mag}$ [40], the peak absolute magnitude was $M_{V}=-17.4$. This is comparable to SN 1997ef and fainter than SN 1998bw by almost 2 mag. Since peak brightness depends on the ejected ${ }^{56} \mathrm{Ni}$ mass, SNe 2002ap, 1997ef, and 1994I appear to have synthesized similar amounts of it. Estimates were $\sim 0.07 \mathrm{M}_{\odot}$ for SN 1994I [33] and $0.13 \mathrm{M}_{\odot}$ for SN 1997ef [23]. The ${ }^{56} \mathrm{Ni}$ mass for $\mathrm{SN} 2002 \mathrm{ap}$ is estimated to be $\sim 0.07 \mathrm{M}_{\odot}$, which is similar to that of normal core-collapse SNe such as SNe 1987A and 1994I.

If line width is the distinguishing feature of a "hypernova," then clearly SN 2002ap belongs to this group, as its spectrum resembles that of SN 1997ef much more than that of SN 1994I (Fig. 1a). Line blending in SN 2002ap and SN 1997 ef is comparable. However, individual features that are clearly visible in SN 1994I but completely blended in SN 1997ef can at least be discerned in SN 2002ap (e.g., the Na I-Si II blend near $6000 \AA$ and the Fe II lines 

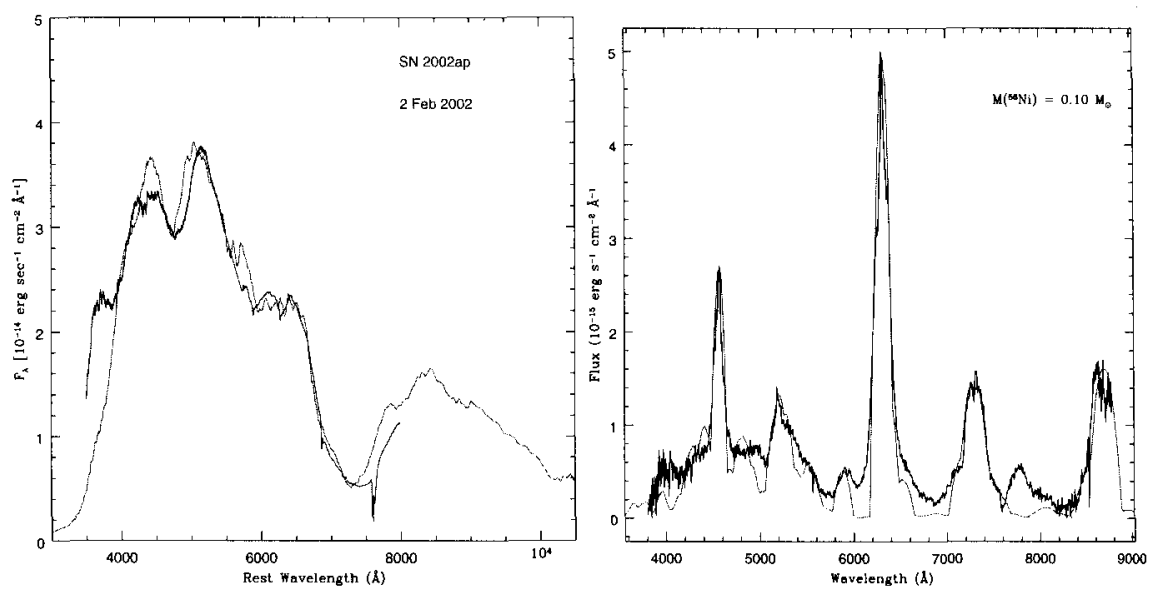

Fig. 2. a. A spectrum of SN 2002ap observed on Feb. 2, 2002, compared to a synthetic spectrum obtained with our Monte Carlo code [24, 22].

b. A spectrum of SN 2002ap observed at Lick Observatory on October 1, 2002, compared to a synthetic NLTE spectrum computed for a ${ }^{56} \mathrm{Ni}$ mass of $0.09 \mathrm{M}_{\odot}$ and a total mass of $\approx 1.3 \mathrm{M}_{\odot}$ below $5600 \mathrm{~km} \mathrm{~s}^{-1}[27]$.

near $5000 \AA$ ). Therefore, spectroscopically SN 2002ap appears to be located just below SN 1997ef in a "velocity scale," but considerably above SN 1994I, confirming the evidence from the light curve.

The spectral evolution of SN 2002ap follows closely that of SN 1997ef, at a rate about 1.5 times faster. The spectra and the light curve of SN 2002ap can be reproduced by a model with $M_{\mathrm{ej}}=2.5-5 \mathrm{M}_{\odot}$ and $E_{51}=4-10$ (see Fig. 2a). Both $M_{\text {ej }}$ and $E_{\text {kin }}$ are much smaller than those of SNe $1998 \mathrm{bw}$ and 1997ef (the higher limits quoted may apply if a significant amount of $\mathrm{He}$ is present).

For these values of $E_{\mathrm{kin}}, M_{\mathrm{ej}}$, and $M\left({ }^{56} \mathrm{Ni}\right)$, we can constrain the progenitor's main-sequence mass $M_{\mathrm{ms}}$ and the remnant mass $M_{\text {rem }}$. Modeling the explosions of $\mathrm{C}+\mathrm{O}$ stars yields $M\left({ }^{56} \mathrm{Ni}\right)$ as a function of the parameter set $\left(E_{\mathrm{kin}}, M_{\mathrm{CO}}, M_{\mathrm{rem}}=M_{\mathrm{ej}}-M_{\mathrm{CO}}\right)$. The model which is most consistent with our estimates of $\left(M_{\mathrm{ej}}, E\right)$ is one with $M_{\mathrm{CO}} \approx 5 \mathrm{M}_{\odot}, M_{\text {rem }} \approx 2.5 \mathrm{M}_{\odot}$, and $E_{51}=4.2$. The $5 M_{\odot} \mathrm{C}+\mathrm{O}$ core forms in a He core of mass $M_{\alpha}=7 \mathrm{M}_{\odot}$, corresponding to a main-sequence mass $M_{\mathrm{ms}} \approx 20-25 \mathrm{M}_{\odot}$. The $M_{\mathrm{ms}}-M_{\alpha}$ relation depends on convection and metallicity (e.g., [34, 41]).

SN 2002ap was not apparently associated with a GRB. This may be a consequence of the fact that the explosion energy of SN 2002ap is about a factor of 5-10 smaller than that of SN 1998bw, as also indicated by the weak radio signature [1]. The present data show no clear signature of asymmetry, except perhaps for some polarization $[14,18,43]$, which is smaller than that of SN 1998bw. This suggests that the degree of asphericity is smaller in SN 2002ap and that the possible "jet" may have been weaker, which makes 

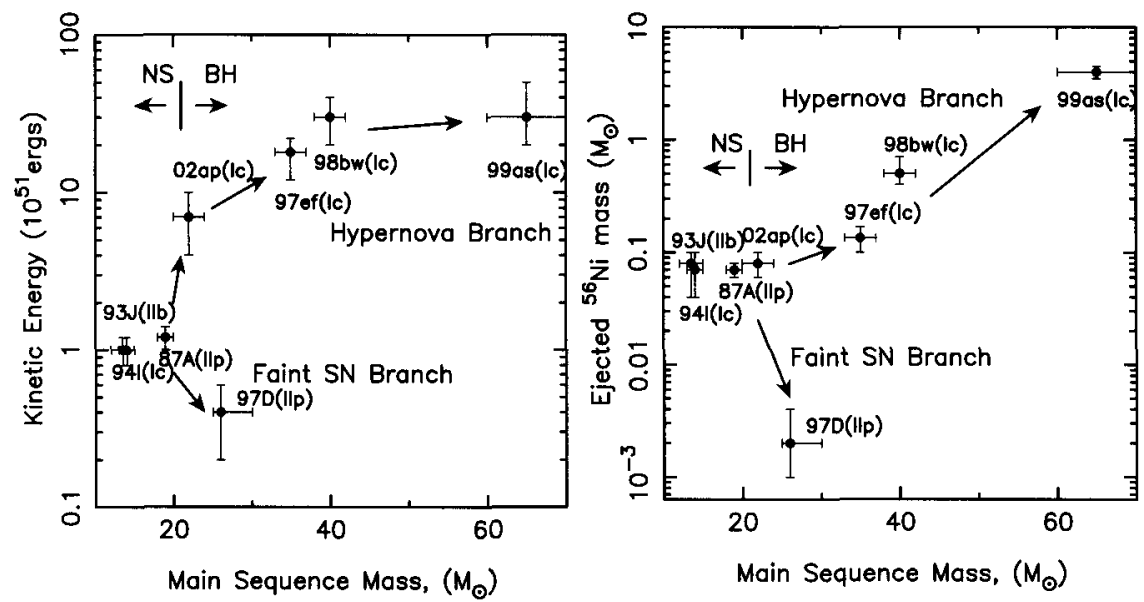

Fig. 3. a. Explosion energies and b. ejected ${ }^{56} \mathrm{Ni}$ mass against main sequence mass of the progenitors for several core collapse supernovae/hypernovae [35].

GRB generation more difficult. It is also possible that a GRB did occur, but that it was not observed because of an unfavorable inclination with respect to the line-of-sight. Asymmetric models indeed show more "normal" properties away from the jet axis.

Nebular spectra, which developed at an epoch of about 4 months, do not show the peculiar narrow [O I] $6300 \AA$ line as in SN 1998bw, but they do show a narrow core in both $\left[\mathrm{O}_{\mathrm{I}}\right]$ and $\left.\mathrm{Mg} \mathrm{I}\right] 4571 \AA$, which may be interpreted as a signature of asymmetry. The mass of ${ }^{56} \mathrm{Ni}$ estimated from modeling these spectra with a $1 \mathrm{D}$ NLTE code (Fig. $2 \mathrm{~b}$ ) is $\approx 0.1 \mathrm{M}_{\odot}$.

\section{Properties of Hypernovae}

Based on the observed objects and their interpretation, it is possible to make some generalization regarding the properties of hyperenergetic type Ic SNe and their relation to the progenitor stars.

\subsection{The Kinetic Energy}

All hypernovae are highly energetic. Fig. 3 shows $E_{\text {kin }}$ as a function of the main-sequence mass $M_{\text {ms }}$ of the progenitor star as derived from fitting the optical light curves and spectra of normal and highly energetic SNe. In a "hypernova branch" $E_{\text {kin }}$ appears to increase with $M_{\mathrm{ms}}$, reaching values much larger than the canonical $10^{51} \mathrm{erg}$. The faint Type II-P SNe $1997 \mathrm{D}$ and $1999 \mathrm{br}$, on the contrary, have very small values of $E_{\text {kin }}$.

This trend might be interpreted as follows. Stars with $M_{\mathrm{ms}} \lesssim 20-25 \mathrm{M}_{\odot}$ form neutron stars and become normal supernovae, their type depending on 
their envelope properties. (SN 1987A may be a borderline case between neutron star and black hole formation.) Stars with $M_{\mathrm{ms}} \gtrsim 20-25 \mathrm{M}_{\odot}$ form black holes (e.g., [4]); whether they become hypernovae or faint SNe may depend on the angular momentum in the collapsing core, which in turn depends on the stellar winds, metallicity, magnetic fields, and binarity.

Hypernova progenitors may have rapidly rotating cores owing possibly to the spiraling-in of a companion star in a binary system. The cores of faint SNe II-P might not have a large angular momentum, because the progenitor's massive H-rich envelope may have been able to transport the angular momentum of the core out to the envelope possibly via a magnetic-field effect.

Between these two branches, there may be a variety of SNe. A dispersion in the properties of SNe II-P has been reported [8], but whether the region of normal explosion energy and large progenitor mass is actually populated remains an open issue awaiting observational verification.

\subsection{The Mass of Ejected ${ }^{56} \mathrm{Ni}$}

A similar relation is observed between the mass of ${ }^{56} \mathrm{Ni}, M\left({ }^{56} \mathrm{Ni}\right)$, synthesized in core-collapse supernovae and $M_{\mathrm{ms}}$ in Fig. 3a. This is important for the study of the chemical evolution of galaxies. Stars with $M_{\mathrm{ms}} \lesssim 20-25 \mathrm{M}_{\odot}$, forming a neutron star, produce $\sim 0.08 \pm 0.03 \mathrm{M}_{\odot}{ }^{56} \mathrm{Ni}$ as in SNe 1993J, 1994I, and 1987A. For stars with $M_{\mathrm{ms}} \gtrsim 20-25 \mathrm{M}_{\odot}$, which form black holes, $M\left({ }^{56} \mathrm{Ni}\right)$ appears to increase with $M_{\mathrm{ms}}$ in the "hypernova branch," while $\mathrm{SNe}$ in the "faint SN branch" produce only very little ${ }^{56} \mathrm{Ni}$. For faint $\mathrm{SNe}$ the explosion energy is so small, probably because of the large gravitational potential, that most ${ }^{56} \mathrm{Ni}$ falls back onto the compact remnant.

\subsection{Asymmetry}

All hyperenergetic SNe Ic show some signatures of asymmetry, or at least of a departure from purely 1-dimensional spherically symmetric models. This may support the case for their connection with at least some GRBs.

Only for SN 1998bw and the recent SN 2003dh/GRB 030329 is the connection with a GRB well established. In the other cases, either a GRB was not generated or, if it was, it was weak and/or not pointing toward us. The issue of directionality is very important. If hypernovae are aspherical, objects with the same ${ }^{56} \mathrm{Ni}$ mass should show a range of properties, reflecting different orientations. These objects may look very different at early phases, displaying different light curves, velocities, and abundances. At late times, these effects should disappear. So far, however, this evidence is missing. Further observations of supernovae in GRBs are obviously essential (e.g., [3, 39]).

\section{References}

1. E. Berger, S.R. Kulkarni, D.A. Frail: IAUC 7817 (2002) 
2. D. Branch: Supernovae and Gamma-Ray Bursts (Proc. of the STScI Symp., Baltimore), eds. M. Livio, N. Panagia, K. Sahu (Cambridge, 2001), pp. 96-109

3. M. Della Valle et al. : Astron. Astrophys. 406, L33 (2003)

4. E. Ergma, E.P.J. van den Heuvel: Astron. Astrophys. 331, L29 (1998)

5. A.V. Filippenko, R. Chornock: IAUC 7825 (2002)

6. T. Galama et al. : Nature 395, 670 (1998)

7. A. Gal-Yam, E.O. Ofek, O. Shemmer: Mon. Not. R. Astron. Soc. 332, L73 (2002)

8. M. Hamuy: Astrophys. J. 582, 905 (2003)

9. Y. Hirose: IAUC 7810 (2002)

10. P. Höflich, J.C. Wheeler, L. Wang: Astrophys. J. 521, 179 (1999)

11. K. Iwamoto et al. : Nature 395, 672 (1998)

12. K. Iwamoto et al. : Astrophys. J. 534, 660 (2000)

13. K. Iwamoto et al. : SN 1998bw and Hypernovae. In: Supernovae and GammaRay Bursters, LNP 598, ed by K. Weiler (Springer, Berlin, Heidelberg, New York, 2003) pp. 243-282

14. K.S. Kawabata et al. : Astrophys. J. Lett. 580, L39 (2002)

15. L.E. Kay, J.P. Halpern, K.M. Leighly, S. Heathcote, A.M. Magalhaes, A.V. Filippenko: IAUC 6969 (1998)

16. K. Kinugasa et al. : IAUC 7811 (2002)

17. R. Knop et al. : IAUC 7128 (1999)

18. D. Leonard, A.V. Filippenko, R. Chornock, R. Foley: Pub. Astron. Soc. Pacific $114,1333(2002)$

19. A.I. MacFadyen, S.E. Woosley: Astrophys. J. 524, 262 (1999)

20. K. Maeda, T. Nakamura, K. Nomoto, P.A. Mazzali, F. Patat, I. Hachisu: Astrophys. J. 565, 405 (2002)

21. T. Matheson, A.V. Filippenko, W. Li, D.C. Leonard, \& J.C. Shields: Astron. J. 121, 1648 (2001)

22. P.A. Mazzali: Astron. Astrophys. 363, 705 (2000)

23. P.A. Mazzali, K. Iwamoto, K. Nomoto: Astrophys. J. 545, 407 (2000)

24. P.A. Mazzali, L.B. Lucy: Astron. Astrophys. 279, 447 (1993)

25. P.A. Mazzali, K. Nomoto, F. Patat, K. Maeda: Astrophys. J. 559, 1047 (2001)

26. P.A. Mazzali et al. : Astrophys. J. Lett. 572, L61 (2002)

27. P.A. Mazzali, K. Nomoto, J. Deng, K. Maeda, Y. Qiu: From Highlight to Twilight: the Physics of Supernovae (ESO, Garching, 2003), p. 246

28. P.A. Mazzali et al. : in preparation

29. P. Meikle et al. : IAUC 7811 (2002)

30. T. Nakamura, P.A. Mazzali, K. Nomoto, K. Iwamoto: Astrophys. J. 550, 991 (2001)

31. T. Nakamura, H. Umeda, K. Iwamoto, K. Nomoto, M. Hashimoto, W.R. Hix, F.-K. Thielemann: Astrophys. J. 555, 880 (2001)

32. S. Nakano, R. Kushida, W. Li: IAUC 7810 (2002)

33. K. Nomoto et al. : Nature 371, 227 (1994)

34. K. Nomoto, M. Hashimoto: Phys. Rep. 256, 173 (1988)

35. K. Nomoto et al. : Supernovae and Gamma-Ray Bursts (Baltimore), eds. M. Livio, N. Panagia, K. Sahu (Cambridge, 2001), pp. 144-170

36. F. Patat et al. : Astrophys. J. 555, 917 (2001)

37. M.W. Richmond et al. : Astron. J. 111, 327 (1996)

38. M.E. Sharina, I.D. Karachentsev, N.A. Tikhonov: Astron. Astrophys. Suppl. 119, 499 (1996) 
39. K.Z. Stanek et al. : Astrophys. J. Lett. 591, L17 (2003)

40. M. Takada-Hidai, W. Aoki, G. Zhao: Pub. Astron. Soc. Japan 54, 899 (2002)

41. H. Umeda, K. Nomoto: Astrophys. J. 565, 385 (2002)

42. L. Wang, J.C. Wheeler: Astrophys. J. Lett. 504, L87 (1998)

43. L. Wang et al. : Astrophys. J. 592, 457 (2003)

44. S. Woosley, R. Eastman, B. Schmidt: Astrophys. J. 516, 788 (1999) 

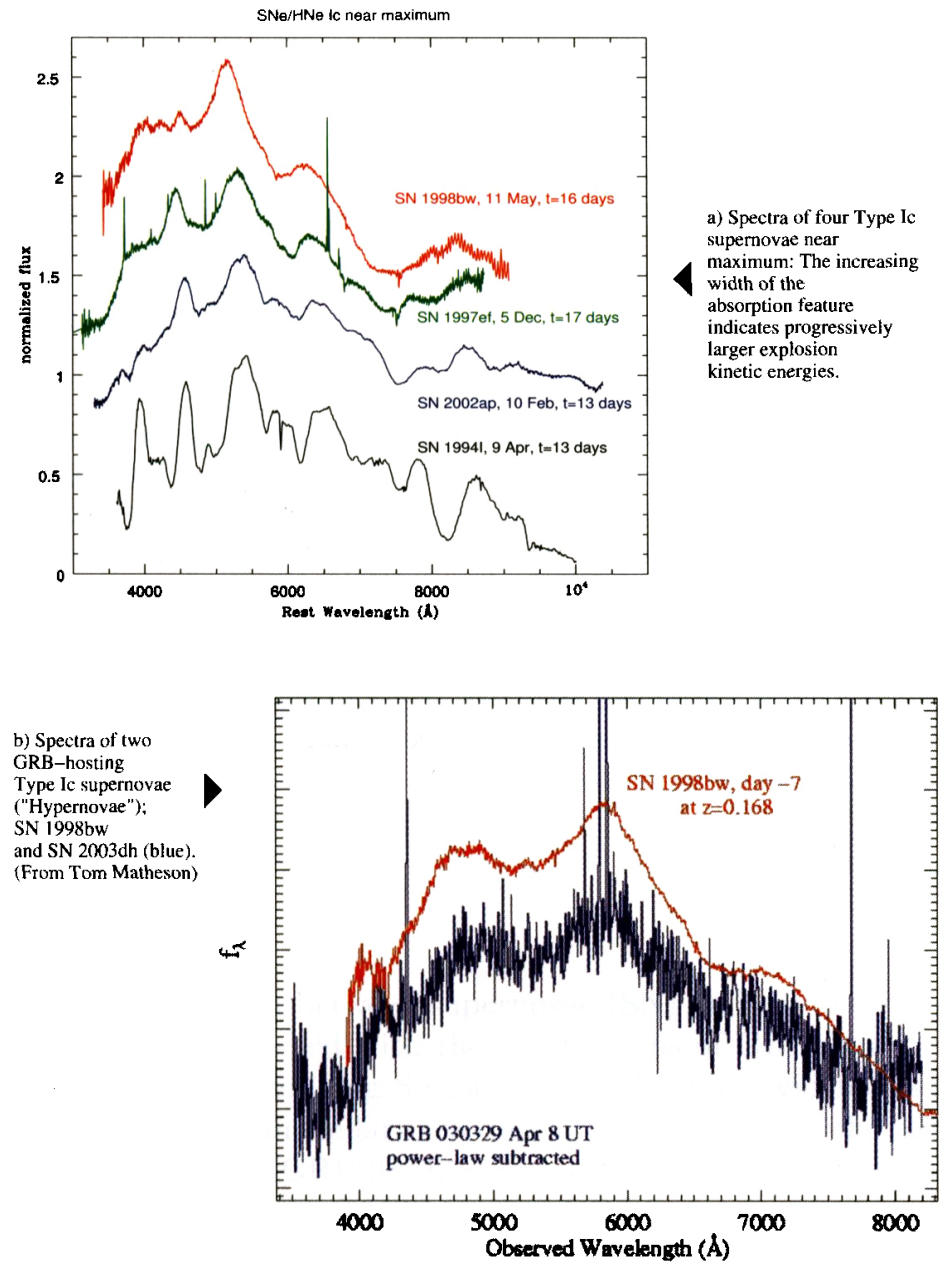

Plate (Mazzali et al.)

Plate 4. 